\title{
Enhanced Higgs mediated lepton flavour violating processes in the supersymmetric inverse seesaw model
}

\author{
Asmaa Abada, Debottam Das and Cédric Weiland \\ Laboratoire de Physique Théorique, CNRS-UMR 8627, Université Paris-Sud 11, \\ F-91405 Orsay Cedex, France \\ E-mail: Asmaa.Abada@th.u-psud.fr, Debottam.Das@th.u-psud.fr, \\ cedric.weiland@th.u-psud.fr
}

ABSTRACT: We study the impact of the inverse seesaw mechanism on several low-energy flavour violating observables such as $\tau \rightarrow \mu \mu \mu$ in the context of the Minimal Supersymmetric Standard Model. As a consequence of the inverse seesaw, the contributions of the right-handed sneutrinos significantly enhance the Higgs-mediated penguin diagrams. We find that different flavour violating branching ratios can be enhanced by as much as two orders of magnitude. We also comment on the impact of the Higgs-mediated processes on the leptonic $B$-meson decays and on the Higgs flavour violating decays.

Keywords: Rare Decays, Beyond Standard Model, Neutrino Physics, Supersymmetric Standard Model

ARXIV EPRINT: 1111.5836 


\section{Contents}

1 Introduction 1

2 Inverse seesaw mechanism in the MSSM 3

3 Lepton flavour violation: Higgs-mediated contributions 5

4 Higgs-mediated lepton flavour violating observables $\quad 8$

5 Results and discussion $\quad 11$

6 Conclusions 13

\section{Introduction}

Neutrino oscillations have provided indisputable evidence for flavour violation in the neutral lepton sector. In the absence of any fundamental principle that prevents charged lepton flavour violation, one expects that extensions of the Standard Model (SM) accommodating neutrino masses and mixings should also allow for lepton flavour violation (LFV) in the charged lepton sector. Indeed, the additional new flavour dynamics and new field content present in many extensions of the SM may provide contributions to charged LFV (cLFV) processes such as radiative (e.g. $\mu \rightarrow e \gamma$ ) and three-body lepton decays (for instance $\tau \rightarrow$ $\mu \mu \mu)$. These decays generally arise from higher order processes, and so their branching ratios (Brs) are expected to be small, making them difficult to observe. Thus, any cLFV signal would provide clear evidence for new physics: mixings in the lepton sector and probably the presence of new particles, possibly shedding light on the origin of neutrino mass generation.

The search for manifestations of charged LFV constitutes the goal of several experiments [1-13], dedicated to look for signals of processes such as rare radiative decays, three-body decays and muon-electron conversion in nuclei. Despite the fact that a cLFV signal could provide clear evidence for new physics, the underlying mechanism of lepton mixing might be difficult to unravel. In parallel to the low-energy searches for new physics, i.e. via indirect effects of possible new particles, the LHC has started to search directly for these new particles in its quest to unveil the mechanism of electroweak symmetry breaking, thus possibly providing a solution to the SM hierarchy problem.

Among the many possible extensions of the SM, supersymmetry (SUSY) is a well motivated solution for the hierarchy problem, providing many other appealing aspects such as gauge coupling unification and dark matter candidates. If the LHC experiments indeed discover SUSY, it is then extremely interesting to consider supersymmetric models that can also explain neutrino masses and mixings. Furthermore, it is only natural to expect 
that such models might also give rise to cLFV. If SUSY is indeed realised in nature, cLFV (mediated by new sparticles) would provide a new probe to explore the origin of lepton mixings, playing a complementary rôle to other searches of new physics, i.e. LHC direct searches and neutrino dedicated experiments.

One of the most economical possibilities is to embed a seesaw mechanism [14-27] in the framework of SUSY models (i.e. the SUSY seesaw) [28, 29]. For any seesaw realisation, the neutrino Yukawa couplings could leave their imprints in the SUSY soft-breaking slepton mass matrices, and consequently induce flavour violation at low energies due to the renormalisation group (RG) evolution of the SUSY soft-breaking parameters. The caveat of these scenarios is that, in order to have sufficiently large Yukawa couplings (as required to account for large cLFV Brs), the typical scale of the extra particles (such as right handed neutrinos, scalar or fermionic isospin triplets) is in general very high, potentially very close to the gauge coupling unification scale. However, such a high (seesaw) scale would be impossible to probe experimentally.

On the other hand, the so-called inverse seesaw [30] constitutes a very appealing alternative to the "standard" seesaw realisations. Embedding an inverse seesaw mechanism in the Minimal Supersymmetric extension of the SM (MSSM) requires the inclusion of two additional gauge singlet superfields, with opposite lepton numbers $(+1$ and -1$)$. When compared to other SUSY seesaw realisations, cLFV observables are enhanced in this framework, and such an enhancement can be attributed to large neutrino Yukawa couplings $\left(Y_{\nu} \sim O(1)\right)$, compatible with a seesaw scale $M$, close to the electroweak one, thus within LHC reach.

The differences between the inverse seesaw and the standard one can be conceptually understood from an effective point of view and linked to the distinct properties of the lepton number violating dimension-5 (Weinberg) operator (responsible for neutrino masses and mixings) and the total lepton number conserving dimension- 6 operator, which is at the origin of cLFV. Contrary to what occurs in the standard seesaw, these two operators are de-correlated in the inverse seesaw, implying that the suppression of the coefficient of the dimension-5 operator will not affect the size of the coefficient of the dimension- 6 operator. In both seesaws, the latter operator is proportional to $\left(Y_{\nu}^{\dagger} \frac{1}{|M|^{2}} Y_{\nu}\right)$; however, in the case of a type I seesaw, the dimension-5 operator is proportional to $\left(Y_{\nu}^{\dagger} \frac{1}{M} Y_{\nu}\right)$, while in the case of an inverse seesaw, it has a further suppression of $\frac{\mu}{M}$ ( $\mu$ being a dimensionful parameter, linked to the mass of the sterile singlets). The dimension- 6 operator will thus be extremely suppressed in the case of a type I seesaw, since in this case $M$ is very large to accommodate natural $Y_{\nu}$. In contrast, in the inverse seesaw, small neutrino masses can easily be accommodated via tiny values of $\mu$, which will not affect the dimension 6 operator. Furthermore, such small values of $\mu$ are natural in the sense of 't Hooft since in the limit where $\mu \rightarrow 0$, the total lepton number symmetry is restored [31].

In view of the strong potential of the inverse seesaw mechanism regarding cLFV, several phenomenological studies have recently been carried out [32-41]. While a nonsupersymmetric inverse seesaw requires two pairs of singlets to explain neutrino oscillation data [38], the supersymmetric generalization can accommodate neutrino data [40] with 
just one pair of singlets. The latter scenario is also known as the minimal supersymmetric inverse seesaw model (MSISM). This model can also comply with the dark matter relic abundance of the Universe [35].

The extra TeV scale singlet neutrinos may significantly contribute to cLFV observables, irrespective of the supersymmetric states [42-45]. Supersymmetric realisations of the inverse seesaw may enhance these cLFV rates even further (e.g. the contributions to $l_{i} \rightarrow l_{j} \gamma$, which has been analysed in [32]). Furthermore, this seesaw model can have LHC signatures: the extra singlets can participate in the decay chains, leading to effects which can be important, particularly in the case in which one of the singlets is the lightest supersymmetric particle (LSP) [40].

In this paper, we focus on contributions to cLFV observables, such as $\tau \rightarrow \mu \mu \mu$, arising from a Higgs-mediated effective vertex. We explore the contributions which are due to the presence of comparatively light right-handed neutrinos and sneutrinos (which are usually negligible in the framework of a type I SUSY-seesaw), while still having large neutrino Yukawa couplings. We find that all these contributions can lead to a significant enhancement of several cLFV observables.

The paper is organised as follows. In section 2, we define the model, providing a brief overview on the implementation of the inverse seesaw in the MSSM. In section 3, we discuss the implications of this model regarding low-energy cLFV observables, particular emphasis being given to the Higgs-mediated processes. In section 4, we study the Higgs-mediated contributions to several lepton flavour violating observables and compare our results to present bounds and to future experimental sensitivities in section 5. Then we draw some generic conclusions on the viability of an inverse seesaw as the underlying mechanism of LFV. We finally conclude in section 6 .

\section{Inverse seesaw mechanism in the MSSM}

The model consists of the MSSM extended by three pairs of singlet superfields, $\widehat{\nu}_{i}^{c}$ and $\widehat{X}_{i}$ $(i=1,2,3)^{1}$ with lepton numbers assigned to be -1 and +1 , respectively. The supersymmetric inverse seesaw model is defined by the superpotential

$$
\begin{aligned}
\mathcal{W}=\varepsilon_{a b}[ & Y_{d}^{i j} \widehat{D}_{i} \widehat{Q}_{j}^{b} \widehat{H}_{d}^{a}+Y_{u}^{i j} \widehat{U}_{i} \widehat{Q}_{j}^{a} \widehat{H}_{u}^{b}+Y_{e}^{i j} \widehat{E}_{i} \widehat{L}_{j}^{b} \widehat{H}_{d}^{a} \\
& \left.+Y_{\nu}^{i j} \widehat{\nu}_{i}^{c} \widehat{L}_{j}^{a} \widehat{H}_{u}^{b}-\mu \widehat{H}_{d}^{a} \widehat{H}_{u}^{b}\right]+M_{R_{i}} \widehat{\nu}_{i}^{c} \widehat{X}_{i}+\frac{1}{2} \mu_{X_{i}} \widehat{X}_{i} \widehat{X}_{i},
\end{aligned}
$$

where $i, j=1,2,3$ denote generation indices. In the above, $\widehat{H}_{d}$ and $\widehat{H}_{u}$ are the down- and up-type Higgs superfields, $\widehat{L}_{i}$ denotes the $\mathrm{SU}(2)$ doublet lepton superfields. $M_{R_{i}}$ represents the right-handed neutrino mass term which conserves lepton number. Due to the presence of non-vanishing $\mu_{X_{i}}$, the total lepton number $L$ is no longer a good quantum number; nevertheless, notice that in our formulation $(-1)^{L}$ is still a good symmetry. Without loss of generality, the terms $\widehat{\nu}_{i}^{c} \widehat{X}_{i}$ and $\widehat{X}_{i} \widehat{X}_{i}$ are taken to be diagonal in generation space. Clearly, as $\mu_{X_{i}} \rightarrow 0$, lepton number conservation is restored, since $M_{R}$ does not violate

$$
{ }^{1} \widetilde{\nu}^{c}=\widetilde{\nu}_{R}^{*} .
$$


lepton number. Although in the present study we consider three generations of $\widehat{\nu}^{c}$ and $\widehat{X}$, we recall that in the minimal version of the SUSY inverse seesaw (where only one generation of $\widehat{\nu}^{c}$ and $\widehat{X}$ is included), neutrino data can be accommodated [40].

The soft SUSY breaking Lagrangian can be written as

$$
\begin{aligned}
-\mathcal{L}_{\text {soft }}= & -\mathcal{L}_{\text {soft }}^{\mathrm{MSSM}}+m_{\widetilde{\nu}^{c}}^{2} \widetilde{\nu}_{i}^{c \dagger} \widetilde{\nu}_{i}^{c}+m_{X}^{2} \widetilde{X}_{i}^{\dagger} \widetilde{X}_{i} \\
& +\left(A_{\nu} Y_{\nu}^{i j} \varepsilon_{a b} \widetilde{\nu}_{i}^{c} \widetilde{L}_{j}^{a} H_{u}^{b}+B_{M_{R_{i}}} \widetilde{\nu}_{i}^{c} \widetilde{X}_{i}+\frac{1}{2} B_{\mu_{X_{i}}} \widetilde{X}_{i} \widetilde{X}_{i}+\text { h.c. }\right),
\end{aligned}
$$

where $\mathcal{L}_{\text {soft }}^{\mathrm{MSSM}}$ denotes the soft SUSY breaking terms of the MSSM. In the above, the singlet scalar states $\widetilde{X}_{i}$ and $\widetilde{\nu}_{i}^{c}$ are assumed to have flavour universal masses, i.e. $m_{X_{i}}^{2}=m_{X}^{2}$ and $m_{\widetilde{\nu}_{i}^{c}}^{2}=m_{\widetilde{\nu}^{c}}^{2}$. The parameters $B_{M_{R_{i}}}$ and $B_{\mu_{X_{i}}}$ are the new terms involving the scalar partners of the sterile neutrino states (notice that while the former conserves lepton number, the latter gives rise to a lepton number violating $\Delta L=2$ term). Working under the assumption of a flavour-blind mechanism for SUSY breaking, we will assume universal boundary conditions ${ }^{2}$ for the soft SUSY breaking parameters at some very high energy scale (e.g. the gauge coupling unification scale $\sim 10^{16} \mathrm{GeV}$ ),

$$
m_{\phi}=m_{0}, \quad M_{\text {gaugino }}=M_{1 / 2}, \quad A_{i}=A_{0} .
$$

Before addressing neutrino mass generation, a few comments on the nature of the superpotential are in order. As can be seen from eq. (2.1), the two singlets $\widehat{\nu}_{i}^{c}$ and $\widehat{X}_{i}$ are differently treated in the sense that a $\Delta L=2$ Majorana mass term is present for $\widehat{X}_{i}$ $\left(\mu_{X_{i}} \widehat{X}_{i} \widehat{X}_{i}\right)$, while no $\mu_{\nu_{i}^{c}} \widehat{\nu}_{i}^{c} \widehat{\nu}_{i}^{c}$ is present in $\mathcal{W}$. Although a generic superpotential with $(-1)^{L}$ should contain the latter term, let us notice that similar to what occurs for $\mu_{X_{i}}$, the absence of $\mu_{\nu_{i}^{c}}$ also enhances the symmetry of the model; moreover, we emphasise that it is the magnitude of $\mu_{X_{i}}$ (and not that of $\mu_{\nu_{i}^{c}}$ ) which controls the size of the light neutrino mass $[36,41]$. In view of this, and for the sake of simplicity, we have assumed $\mu_{\nu_{i}^{c}}=0$ (considering non-vanishing, yet small values of $\mu_{\nu_{i}^{c}}$ would not change the qualitative features of the model). Although in our formulation we treat $\mu_{X_{i}}$ as an effective parameter, its origin can be explained either dynamically or in a framework of SUSY Grand Unified Theories (GUT) [36, 37, 41]. Furthermore $\mu_{\nu_{i}^{c}} \ll \mu_{X_{i}}$ can also be realised in extended frameworks [36].

In order to illustrate the pattern of light neutrino masses in the inverse seesaw model and how it is related to the lepton number violating parameter $\mu_{X_{i}}$, we consider the onegeneration case. In the $\left\{\nu, \nu^{c}, X\right\}$ basis the $(3 \times 3)$ neutrino mass matrix can be written as

$$
\mathcal{M}=\left(\begin{array}{ccc}
0 & m_{D} & 0 \\
m_{D} & 0 & M_{R} \\
0 & M_{R} & \mu_{X}
\end{array}\right)
$$

\footnotetext{
${ }^{2}$ In our subsequent numerical analysis, we will relax some of these universality conditions, considering non-universal soft breaking terms for the Higgs sector. In what concerns the right-handed sneutrino sector, we will assume that the corresponding soft-breaking masses hardly run between the GUT and the low-energy scale.
} 
with $m_{D}=Y_{\nu} v_{u}$, yielding the mass eigenvalues $\left(m_{1} \ll m_{2,3}\right)$ :

$$
m_{1}=\frac{m_{D}^{2} \mu_{X}}{m_{D}^{2}+M_{R}^{2}}, \quad m_{2,3}=\mp \sqrt{M_{R}^{2}+m_{D}^{2}}+\frac{M_{R}^{2} \mu_{X}}{2\left(m_{D}^{2}+M_{R}^{2}\right)} .
$$

The above equation clearly reveals that the lightness of the smallest eigenvalue $m_{1}$ is indeed due to the smallness of $\mu_{X}\left(\mu_{X} \simeq m_{1}\right)$. Thus the lepton number conserving mass parameters $\left(m_{D}\right.$ and $\left.M_{R}\right)$ are completely unconstrained in this model. Finally, it is worth noticing that the effective right-handed sneutrino mass term (Dirac-like) is given by $M_{\widetilde{\nu}_{i}^{c}}^{2}=$ $m_{\widetilde{\nu}^{c}}^{2}+M_{R_{i}}^{2}+\sum_{j}\left|Y_{\nu}^{i j}\right|^{2} v_{u}^{2}$. Assuming $M_{R} \sim \mathcal{O}(\mathrm{TeV})$, the effective mass term will not be very large, in clear contrast to what occurs in the standard (type I) SUSY seesaw. In our analysis, we will be particularly interested in the rôle of such a light sneutrino (i.e. $\left.M_{\widetilde{\nu}^{c}}^{2} \sim M_{\text {SUSY }}^{2}\right)$ in the enhancement of Higgs mediated contributions to lepton flavour violating observables.

\section{Lepton flavour violation: Higgs-mediated contributions}

In the SUSY seesaw framework, the only source of flavour violation is encoded in the neutrino Yukawa couplings (which are necessarily non-diagonal to account for neutrino oscillation data); even under the assumption of universal soft breaking terms at the GUT scale, radiative effects proportional to $Y_{\nu}$ induce flavour violation in the slepton mass matrices, which in turn give rise to slepton mediated cLFV observables [46-48]. As an example, in the leading logarithmic approximation, the RGE corrections to the left-handed slepton soft-breaking masses are given by

$$
\begin{aligned}
\left(\Delta m_{\widetilde{L}}^{2}\right)_{i j} & \simeq-\frac{1}{8 \pi^{2}}\left(3 m_{0}^{2}+A_{0}^{2}\right)\left(Y_{\nu}^{\dagger} L Y_{\nu}\right)_{i j}, \quad L=\ln \frac{M_{\mathrm{GUT}}}{M_{R}} \\
& =\xi\left(Y_{\nu}^{\dagger} Y_{\nu}\right)_{i j} .
\end{aligned}
$$

(For simplicity, in the above we are implicitly assuming a degenerate right-handed neutrino spectrum, $M_{R_{i}}=M_{R}$.) The RGE-induced flavour violating entries, $\left(\Delta m_{\widetilde{L}}^{2}\right)_{i j}$, give rise to the dominant contributions to low-energy flavour violating observables in the charged lepton sector, such as $\ell_{i} \rightarrow \ell_{j} \gamma$ (mediated by chargino-sneutrino and neutralino-slepton loops) and $\ell_{i} \rightarrow \ell_{j} \ell_{k} \ell_{m}$ (from photon, $Z$ and Higgs mediated penguin diagrams).

Compared to the standard (type I) SUSY seesaw, where $M_{R} \sim 10^{14} \mathrm{GeV}$, the inverse seesaw is characterised by a right handed neutrino mass scale $M_{R} \sim \mathcal{O}(\mathrm{TeV})$ and this in turn leads to an enhancement of the factor $\xi$, (see eq. (3.1)), and hence to all low-energy cLFV observables, in the latter framework. Furthermore, having right-handed sneutrinos whose mass is of the same order of the other sfermions, i.e. $M_{\widetilde{\nu}^{c}}^{2} \sim M_{\mathrm{SUSY}}^{2}$, the $\widetilde{\nu}^{c}$-mediated processes are no longer suppressed, and might even significantly contribute to the lowenergy flavour violating observables. Here, we focus on the impact of such a light $\widetilde{\nu}^{c}$ in the Higgs mediated processes which are expected to be important in the large $\tan \beta$ regime.

Although at tree level Higgs-mediated neutral currents are flavour conserving, nonholomorphic Yukawa interactions of the type $\bar{D}_{R} Q_{L} H_{u}^{*}$ can be induced at the one-loop level, as first noticed in [49]. In the large $\tan \beta$ regime, in addition to providing significant 
corrections to the masses of the $b$-quark, these non-holomorphic couplings have an impact on $B^{0}-\bar{B}^{0}$ mixing and flavour violating decays, in particular $B_{s} \rightarrow \mu^{+} \mu^{-}$[50-57]. Similarly, in the lepton sector, the origin of the Higgs-mediated flavour violating couplings can be traced to a non-holomorphic Yukawa term of the form $\bar{E}_{R} L H_{u}^{*}$ [58]. Other than the corrections to the $\tau$ lepton mass, these new couplings give rise to additional contributions to several cLFV processes mediated by Higgs exchange. In particular $B_{s} \rightarrow \mu \tau, B_{s} \rightarrow e \tau$ (the so-called double penguin processes) were considered in [57], while $\tau \rightarrow \mu \eta$ was studied in [59]. A detailed analysis of the several $\mu-\tau$ lepton flavour violating processes, namely $\tau \rightarrow \mu X\left(X=\gamma, e^{+} e^{-}, \mu^{+} \mu^{-}, \rho, \pi, \eta, \eta^{\prime}\right)$ can be found in [60].

Even though the flavour violating processes in the quark and lepton sectors have a similar diagrammatic origin, the source of flavour violation is different in each case. In the quark sector, trilinear soft SUSY breaking couplings involving up-type squarks provide the dominant source of flavour violation [51], while in the lepton case, LFV stems from the radiatively induced non-diagonal terms in the slepton masses (see eq. (3.1)) [58].

In the standard SUSY seesaw (type I), the term $\widetilde{\nu}_{i}^{c} H_{u} \widetilde{L}_{L j}$ is usually neglected, as it is suppressed by the very heavy right handed sneutrino masses $\left(M_{\widetilde{\nu}_{i}^{c}} \sim 10^{14} \mathrm{GeV}\right)$. However, in scenarios such as the inverse SUSY seesaw, where $M_{\widetilde{\nu}_{i}^{c}} \sim \mathcal{O}(\mathrm{TeV})$, this term may provide the dominant contributions to Higgs mediated lepton flavour violation.

The effective Lagrangian describing the couplings of the neutral Higgs fields to the charged leptons is given by

$$
-\mathcal{L}^{\mathrm{eff}}=\bar{E}_{R}^{i} Y_{e}^{i i}\left[\delta_{i j} H_{d}^{0}+\left(\epsilon_{1} \delta_{i j}+\epsilon_{2 i j}\left(Y_{\nu}^{\dagger} Y_{\nu}\right)_{i j}\right) H_{u}^{0 *}\right] E_{L}^{j}+\text { h.c. } .
$$

In the above, the first term corresponds to the usual Yukawa interaction, while the coefficient $\epsilon_{1}$ encodes the corrections to the charged lepton Yukawa couplings. In the basis where the charged lepton Yukawa couplings are diagonal, the last term in eq. (3.2), i.e. $\epsilon_{2 i j}\left(Y_{\nu}^{\dagger} Y_{\nu}\right)_{i j}$, is in general non-diagonal, thus providing a new source of charged lepton flavour violation through Higgs mediation. Its origin can be diagrammatically understood from figure 1 , where flavour violation is parametrized via a mass insertion $\left(\Delta m_{\widetilde{L}}^{2}\right)_{i j}$ (see eq. (3.1)). The coefficient $\epsilon_{2}$ can be estimated as

$$
\begin{aligned}
\epsilon_{2 i j}= & \frac{\alpha^{\prime}}{8 \pi} \xi \mu M_{1}\left[2 F_{2}\left(M_{1}^{2}, m_{\widetilde{E}_{L j}}^{2}, m_{\widetilde{E}_{L i}}^{2}, m_{\widetilde{E}_{R i}}^{2}\right)-F_{2}\left(\mu^{2}, m_{\widetilde{E}_{L j}}^{2}, m_{\widetilde{E}_{L i}}^{2}, M_{1}^{2}\right)\right] \\
& +\frac{\alpha_{2}}{8 \pi} \xi \mu M_{2}\left[F_{2}\left(\mu^{2}, m_{\widetilde{E}_{L j}}^{2}, m_{\widetilde{E}_{L i}}^{2}, M_{2}^{2}\right)+2 F_{2}\left(\mu^{2}, m_{\widetilde{\nu}_{L j}}^{2}, m_{\widetilde{\nu}_{L i}}^{2}, M_{2}^{2}\right)\right]
\end{aligned}
$$

where

$$
F_{2}(x, y, z, w)=-\frac{x \ln x}{(x-y)(x-z)(x-w)}-\frac{y \ln y}{(y-x)(y-z)(y-w)}+(x \leftrightarrow z, y \leftrightarrow w) .
$$

Here, $M_{1}$ and $M_{2}$ are the masses of the electroweak gauginos at low energies. On the other hand, the flavour conserving loop-induced form factor $\epsilon_{1}$ (notice that the diagrams of figure 1 contribute to this form factor, but without the slepton flavour mixings in the internal lines) can be expressed as $[57,58]$

$$
\begin{aligned}
\epsilon_{1}= & \frac{\alpha^{\prime}}{8 \pi} \mu M_{1}\left[2 F_{1}\left(M_{1}^{2}, m_{\widetilde{E}_{L}}^{2}, m_{\widetilde{E}_{R}}^{2}\right)-F_{1}\left(M_{1}^{2}, \mu^{2}, m_{\widetilde{E}_{L}}^{2}\right)+2 F_{1}\left(M_{1}^{2}, \mu^{2}, m_{\widetilde{E}_{R}}^{2}\right)\right] \\
& +\frac{\alpha_{2}}{8 \pi} \mu M_{2}\left[F_{1}\left(\mu^{2}, m_{\widetilde{E}_{L}}^{2}, M_{2}^{2}\right)+2 F_{1}\left(\mu^{2}, m_{\widetilde{\nu}_{L}}^{2}, M_{2}^{2}\right)\right]
\end{aligned}
$$



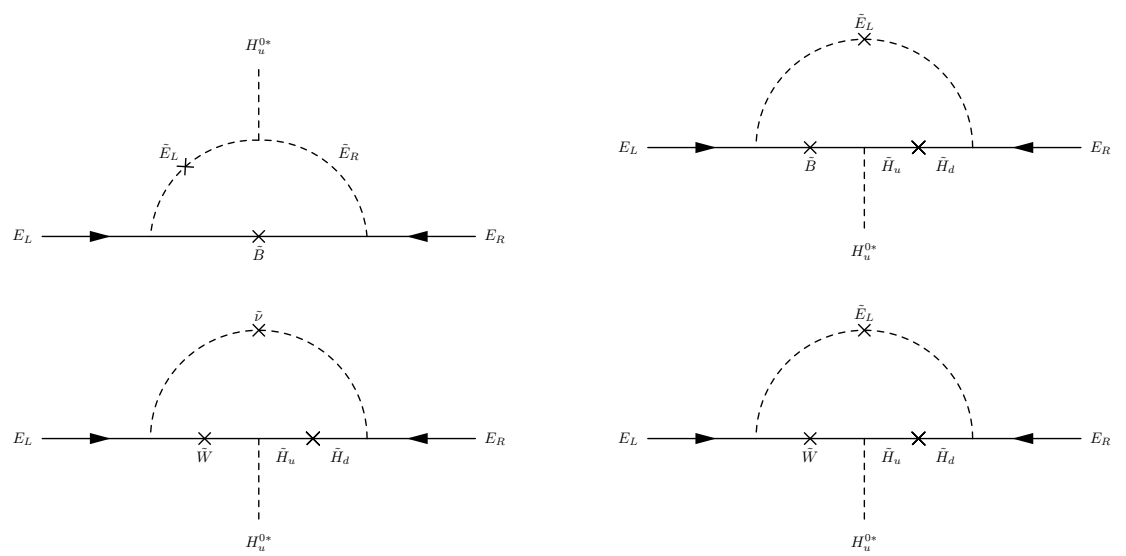

Figure 1. Diagrams contributing to $\epsilon_{2}$. Crosses on scalar lines represent LFV mass insertions $\left(\Delta m_{\widetilde{L}}^{2}\right)_{i j}$, while those on fermion lines denote chirality flips.

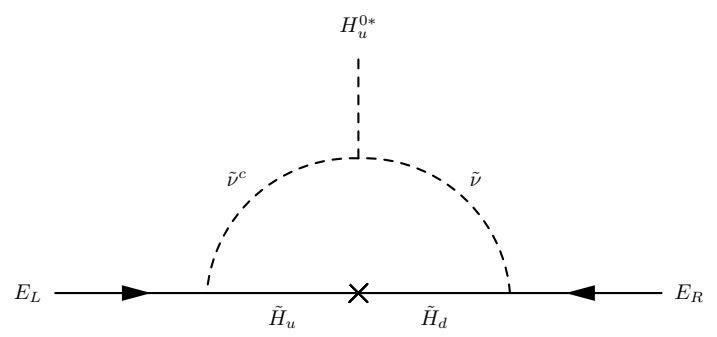

Figure 2. Right-handed sneutrino contribution to $\epsilon_{2}^{\prime}$. This contribution is particularly relevant when $\widetilde{\nu}^{c}$ is light.

with

$$
F_{1}(x, y, z)=-\frac{x y \ln (x / y)+y z \ln (y / z)+z x \ln (z / x)}{(x-y)(y-z)(z-x)} .
$$

In the standard seesaw mechanism, the diagrams of figure 1 provide the only source for Higgs-mediated lepton flavour violation. However, in the framework of the inverse SUSY seesaw, there is an additional diagram that may even account for the dominant Higgsmediated lepton flavour violation contribution: the sneutrino-chargino mediated loop, depicted in figure 2. (Due to the large masses of $\widetilde{\nu}^{c}$ in the standard (type I) seesaw, this process provides negligible contributions, and is hence not taken into account.)

The effective Lagrangian terms encoding lepton flavour violation is accordingly modified as

$$
-\mathcal{L}^{\mathrm{LFV}}=\bar{E}_{R}^{i} Y_{e}^{i i} \epsilon_{2 i j}^{\mathrm{tot}}\left(Y_{\nu}^{\dagger} Y_{\nu}\right)_{i j} H_{u}^{0 *} E_{L}^{j}+\text { h.c. },
$$

where $\epsilon_{2}^{\text {tot }}=\epsilon_{2}+\epsilon_{2}^{\prime}, \epsilon_{2}^{\prime}$ being the contribution from the new diagram. This contribution can be expressed as

$$
\epsilon_{2 i j}^{\prime}=\frac{1}{16 \pi^{2}} \mu A_{\nu} F_{1}\left(\mu^{2}, m_{\widetilde{\nu}_{i}}^{2}, M_{\widetilde{\nu}_{j}^{c}}^{2}\right)
$$

In the above, we have parametrized the soft trilinear term for the neutral leptons as $A_{\nu} Y_{\nu}$, where $A_{\nu}$ is a flavour independent real mass term. 
Below, we provide an approximate estimate of the relative contributions of the terms $\epsilon_{2}$ and $\epsilon_{2}^{\prime}$ : for simplicity we take $M_{\widetilde{\nu}^{c}} \sim \mathcal{O}(\mathrm{TeV})$ and assume common values for the masses of all SUSY particles and dimensionful terms $A_{\nu}$ at low energies, symbolically denoted by $A_{\nu} \sim\langle\widetilde{m}\rangle \sim M_{\mathrm{SUSY}}$. In this limit, the loop functions are given by $F_{2}(x, x, x, x)=\frac{1}{6 x^{2}}$ and $F_{1}(x, x, x)=\frac{1}{2 x}$. This leads to

$$
\epsilon_{2}=\frac{1}{8 \pi} \xi \widetilde{m}^{2}\left(\frac{\alpha^{\prime}}{6 \widetilde{m}^{4}}+3 \frac{\alpha_{2}}{6 \widetilde{m}^{4}}\right) \simeq-0.0007
$$

while

$$
\epsilon_{2}^{\prime}=\frac{1}{16 \pi^{2}} \widetilde{m}^{2} \frac{1}{2 \widetilde{m}^{2}} \simeq 0.003 .
$$

In this illustrative (leading order) calculation, we have assumed that at $M_{\mathrm{GUT}}$, one has $A_{0}=0$, taking for the gauge couplings $\alpha_{2}=0.03$ and $\alpha^{\prime}=0.008$. Following eq. (3.1), and assuming $M_{R}=10^{3} \mathrm{GeV}$, one gets $\xi \sim-1.1 \mathrm{~m}_{0}^{2}$. Thus, at the leading order in the inverse seesaw, the lepton flavour violation coefficient becomes $\left|\epsilon_{2}^{\text {tot }}\right|=\left|\epsilon_{2}+\epsilon_{2}^{\prime}\right| \simeq 2 \times 10^{-3}$.

For completeness, let us notice that in the standard seesaw model (where sizable Yukawa couplings are typically associated to a right-handed neutrino mass scale $\sim 10^{14} \mathrm{GeV}$ ), assuming the same amount of flavour violation as parametrized by $\xi$, one finds $\left|\epsilon_{2}^{\text {tot }}\right|=\left|\epsilon_{2}\right| \simeq 2 \times 10^{-4}$. This clearly reveals that in the inverse SUSY seesaw, $\epsilon_{2}^{\text {tot }}$ is enhanced by a factor of order $\sim 10$ compared to the standard seesaw.

The large enhancement of $\epsilon_{2}^{\text {tot }}$ will have an impact regarding all Higgs-mediated lepton flavour violating observables. The computation of the cLFV observables requires specifying the couplings of the physical Higgs bosons to the leptons, in particular $\bar{E}_{R}^{i} E_{L}^{j} H_{k}$ (where $\left.H_{k}=h, H, A\right)$. The effective Lagrangian describing this interaction can be derived from eq. $(3.2)$, and reads $[57,58]$ as

$$
-\mathcal{L}_{i \neq j}^{\text {eff }}=\left(2 G_{F}^{2}\right)^{1 / 4} \frac{m_{E_{i}} \kappa_{i j}^{E}}{\cos ^{2} \beta}\left(\bar{E}_{R}^{i} E_{L}^{j}\right)[\cos (\alpha-\beta) h+\sin (\alpha-\beta) H-i A]+\text { h.c. },
$$

where $\alpha$ is the CP-even Higgs mixing angle and $\tan \beta=v_{u} / v_{d}$, and

$$
\kappa_{i j}^{E}=\frac{\epsilon_{2 i j}^{\mathrm{tot}}\left(Y_{\nu}^{\dagger} Y_{\nu}\right)_{i j}}{\left[1+\left(\epsilon_{1}+\epsilon_{2 i i}^{\mathrm{tot}}\left(Y_{\nu}^{\dagger} Y_{\nu}\right)_{i i}\right) \tan \beta\right]^{2}} .
$$

As clear from the above equation, large values of $\epsilon_{2}^{\text {tot }}$ lead to an augmentation of $\kappa_{i j}^{E}$. Given that the cLFV branching ratios are proportional to $\left(\kappa_{i j}^{E}\right)^{2}$, a sizeable enhancement, as large as two orders of magnitude, is expected for all Higgs-mediated LFV observables.

\section{Higgs-mediated lepton flavour violating observables}

Here we focus our attention on the cLFV observables where the dominant contribution to flavour violation arises from the Higgs penguin diagrams, in particular those involving $\tau$-leptons (due to the comparatively large value of $Y_{\tau}$ ).

In what follows, we discuss some of these LFV decays in detail. 
- $\tau \rightarrow 3 \mu$

In the large $\tan \beta$ regime, Higgs-mediated flavour violating diagrams would be particularly important in this decay mode. The branching ratio can be expressed as $[57,58]$

$$
\begin{aligned}
\operatorname{Br}(\tau \rightarrow 3 \mu) & =\frac{G_{F}^{2} m_{\mu}^{2} m_{\tau}^{7} \tau_{\tau}}{1536 \pi^{3} \cos ^{6} \beta}\left|\kappa_{\tau \mu}^{E}\right|^{2}\left[\left(\frac{\sin (\alpha-\beta) \cos \alpha}{M_{H}^{2}}-\frac{\cos (\alpha-\beta) \sin \alpha}{M_{h}^{2}}\right)^{2}+\frac{\sin ^{2} \beta}{M_{A}^{4}}\right] \\
& \approx \frac{G_{F}^{2} m_{\mu}^{2} m_{\tau}^{7} \tau_{\tau}}{768 \pi^{3} M_{A}^{4}}\left|\kappa_{\tau \mu}^{E}\right|^{2} \tan ^{6} \beta .
\end{aligned}
$$

In the above, $\tau_{\tau}$ is the $\tau$ life time and the approximate result has been obtained in the large $\tan \beta$ regime. For other Higgs-mediated lepton flavour violating 3-body decays, $\tau \rightarrow e \mu \mu, \tau \rightarrow 3 e$ or $\mu \rightarrow 3 e$, their corresponding branching ratios can easily be obtained with the appropriate kinematic factors and the flavour changing factor $\kappa$. While $\operatorname{Br}(\tau \rightarrow e \mu \mu)$ can be as large as $\operatorname{Br}(\tau \rightarrow 3 \mu)$ when $\left(Y_{\nu}^{\dagger} Y_{\nu}\right)_{13} \sim O(1)$ (which is possible in the case of an inverted hierarchical light neutrino spectrum), other flavour violating decays with final state electrons such as $\mu \rightarrow 3 e$ are considerably suppressed due to the smallness of the Yukawa couplings.

- $B_{s} \rightarrow \ell_{i} \ell_{j}$

$B$ mesons can also have Higgs-mediated LFV decays, which are significantly enhanced in the large $\tan \beta$ regime. The branching fraction is given by

$$
\begin{aligned}
\operatorname{Br}\left(B_{s} \rightarrow \ell_{i} \ell_{j}\right)= & \frac{G_{F}^{4} M_{W}^{4}}{8 \pi^{5}}\left|V_{t b}^{*} V_{t s}\right|^{2} M_{B_{s}}^{5} f_{B_{s}}^{2} \tau_{B_{s}}\left(\frac{m_{b}}{m_{b}+m_{s}}\right)^{2} \\
& \times \sqrt{\left[1-\frac{\left(m_{\ell_{i}}+m_{\ell_{j}}\right)^{2}}{M_{B_{s}}^{2}}\right]\left[1-\frac{\left(m_{\ell_{i}}-m_{\ell_{j}}\right)^{2}}{M_{B_{s}}^{2}}\right]} \\
& \times\left\{\left(1-\frac{\left(m_{\ell_{i}}+m_{\ell_{j}}\right)^{2}}{M_{B_{s}}^{2}}\right)\left|c_{S}^{i j}\right|^{2}+\left(1-\frac{\left(m_{\ell_{i}}-m_{\ell_{j}}\right)^{2}}{M_{B_{s}}^{2}}\right)\left|c_{P}^{i j}\right|^{2}\right\},
\end{aligned}
$$

where $V_{i j}$ represents the Cabibbo-Kobayashi-Maskawa (CKM) matrix, $M_{B_{s}}$ and $\tau_{B_{s}}$ respectively denote the mass and lifetime of the $B_{s}$ meson, while $f_{B_{s}}=$ $230 \pm 30 \mathrm{MeV}$ [61] is the $B_{s}$ meson decay constant and $c_{P}^{i j}, c_{S}^{i j}$ are the form factors. As an example, the lepton flavour violating (double-penguin) $B_{s} \rightarrow \mu \tau$ decay can be computed with the following form factors [57]:

$$
\begin{aligned}
c_{S}^{\mu \tau}=c_{P}^{\mu \tau} & =\frac{\sqrt{2} \pi^{2}}{G_{F} M_{W}^{2}} \frac{m_{\tau} \kappa_{b s}^{d} \kappa_{\tau \mu}^{E *}}{\cos ^{4} \beta \bar{\lambda}_{b s}^{t}}\left[\frac{\sin ^{2}(\alpha-\beta)}{M_{H}^{2}}+\frac{\cos ^{2}(\alpha-\beta)}{M_{h}^{2}}+\frac{1}{M_{A}^{2}}\right] \\
& \approx \frac{8 \pi^{2} m_{\tau} m_{t}^{2}}{M_{W}^{2}} \frac{\epsilon_{Y} \kappa_{\tau \mu}^{E} \tan ^{4} \beta}{\left[1+\left(\epsilon_{0}+\epsilon_{Y} Y_{t}^{2}\right) \tan \beta\right]\left[1+\epsilon_{0} \tan \beta\right]} \frac{1}{M_{A}^{2}} .
\end{aligned}
$$

Here, $\kappa_{b s}^{d}$ represents the flavour mixing in the quark sector while $\bar{\lambda}_{b s}^{t}=V_{t b}^{*} V_{t s}$. Similarly, $\epsilon_{0}$ and $\epsilon_{Y}$ are the down type quark form factors mediated by gluino and squark exchange diagrams. The final result was, once again, derived in the large $\tan \beta$ regime. The branching fractions of other flavour violating decays such as 
$\operatorname{Br}\left(B_{d, s} \rightarrow \tau e\right)$, would receive identical contribution from the Higgs penguins. Likewise, the $\operatorname{Br}\left(B_{d, s} \rightarrow \mu e\right)$ can be calculated using the appropriate form factors and lepton masses; as expected, these will be suppressed when compared to $\operatorname{Br}\left(B_{d, s} \rightarrow \tau \mu\right)$.

- $\tau \rightarrow \mu P$

Similar to what occurred in the previous processes, virtual Higgs exchange could also induce decays such as $\tau \rightarrow \mu P$, where $P$ denotes a neutral pseudoscalar meson $(P=$ $\left.\pi, \eta, \eta^{\prime}\right)$. In the large $\tan \beta$ limit, where the pseudoscalar Higgs couplings to downtype quarks are enhanced, CP-odd Higgs boson exchanges provide the dominant contribution to the $\tau \rightarrow \mu P$ decay. The coupling can be written as

$$
-i\left(\sqrt{2} G_{F}\right)^{1 / 2} \tan \beta A\left(\xi_{d} m_{d} \bar{d} d+\xi_{s} m_{s} \bar{s} s+\xi_{b} m_{b} \bar{b} b\right)+\text { h.c. } .
$$

Here, the parameters $\xi_{d}, \xi_{s}, \xi_{b}$ are of order $\mathcal{O}(1)$. Since we are mostly interested in the Higgs-mediated contributions, we estimate the amplitude of these processes in the limit when both $\tau \rightarrow 3 \mu$ and $\tau \rightarrow \mu P$ are indeed dominated by the exchange of the scalar fields. Accordingly, and following [60], one can write

$$
\begin{aligned}
& \frac{\operatorname{Br}(\tau \rightarrow \mu \eta)}{\operatorname{Br}(\tau \rightarrow 3 \mu)} \simeq 36 \pi^{2}\left(\frac{f_{\eta}^{8} m_{\eta}^{2}}{m_{\mu} m_{\tau}^{2}}\right)^{2}\left(1-x_{\eta}\right)^{2}\left[\xi_{s}+\frac{\xi_{b}}{3}\left(1+\sqrt{2} \frac{f_{\eta}^{0}}{f_{\eta}^{8}}\right)\right]^{2}, \\
& \frac{\operatorname{Br}\left(\tau \rightarrow \mu \eta^{\prime}\right)}{\operatorname{Br}(\tau \rightarrow \mu \eta)} \simeq \frac{2}{9}\left(\frac{f_{\eta^{\prime}}^{0}}{f_{\eta}^{8}}\right)^{2} \frac{m_{\eta^{\prime}}^{4}}{m_{\eta}^{4}}\left(\frac{1-x_{\eta^{\prime}}}{1-x_{\eta}}\right)^{2}\left[\frac{1+\frac{3}{\sqrt{2}} \frac{f_{\eta^{\prime}}^{8}}{f_{\eta^{\prime}}^{0}}\left(\frac{\xi_{s}}{\xi_{b}}+\frac{1}{3}\right)}{\left.\frac{\xi_{s}}{\xi_{b}}+\frac{1}{3}+\frac{\sqrt{2}}{3} \frac{f_{\eta}^{0}}{f_{\eta}^{8}}\right]^{2},}\right. \\
& \frac{\operatorname{Br}(\tau \rightarrow \mu \pi)}{\operatorname{Br}(\tau \rightarrow \mu \eta)} \simeq \frac{4}{3}\left(\frac{f_{\pi}}{f_{\eta}^{8}}\right)^{2} \frac{m_{\pi}^{4}}{m_{\eta}^{4}}\left(1-x_{\eta}\right)^{-2}\left[\frac{\frac{\xi_{d}}{\xi_{b}} \frac{1}{1+z}+\frac{1}{2}\left(1+\frac{\xi_{s}}{\xi_{b}}\right) \frac{1-z}{1+z}}{\frac{\xi_{s}}{\xi_{b}}+\frac{1}{3}+\frac{\sqrt{2}}{3} \frac{f_{\eta}^{0}}{f_{\eta}^{8}}}\right]^{2},
\end{aligned}
$$

where $z=m_{u} / m_{d}, m_{\pi}, f_{\pi}$ are the pion mass and decay constant, $m_{\eta, \eta^{\prime}}$ are the masses of $\eta, \eta^{\prime}, x_{\eta, \eta^{\prime}}=m_{\eta, \eta^{\prime}}^{2} / m_{\pi}^{2}$, and $f_{\eta, \eta^{\prime}}^{8}$ and $f_{\eta, \eta^{\prime}}^{0}$ are evaluated from the corresponding matrix elements. As first discussed in [59], and taking $\xi_{s}, \xi_{b} \sim 1$ and fixing the other parameters as in [60], one finds $\frac{\operatorname{Br}(\tau \rightarrow \mu \eta)}{\operatorname{Br}(\tau \rightarrow 3 \mu)} \simeq 5$. The other branching fractions such as $\operatorname{Br}\left(\tau \rightarrow \mu \eta^{\prime}, \mu \pi\right)$ are considerably suppressed compared to $\operatorname{Br}(\tau \rightarrow \mu \eta)$. While the ratio $\frac{\operatorname{Br}\left(\tau \rightarrow \mu \eta^{\prime}\right)}{\operatorname{Br}(\tau \rightarrow \mu \eta)}$ can be as large as $6 \times 10^{-3}, \frac{\operatorname{Br}(\tau \rightarrow \mu \pi)}{\operatorname{Br}(\tau \rightarrow \mu \eta)}$ would approximately lie in the range $10^{-3}-4 \times 10^{-3}[60]$. Since all these ratios are independent of $\kappa_{\tau \mu}^{E}$, the above quoted numbers can also be applied to the present framework. However, an enhancement in the $\operatorname{Br}(\tau \rightarrow 3 \mu)$, due to the large values of $\kappa_{\tau \mu}^{E}$, would also imply sizeable values of $\operatorname{Br}(\tau \rightarrow \mu \eta)$.

- $H_{k} \rightarrow \mu \tau\left(H_{k}=h, H, A\right)$

The branching ratios of flavour violating Higgs decays provide another interesting probe of lepton flavour violation. Following [62], the branching fraction $H_{k} \rightarrow \mu \tau$ (normalised to the flavour conserving one $H_{k} \rightarrow \tau \tau$ ) can be cast as:

$$
\operatorname{Br}\left(H_{k} \rightarrow \mu \tau\right)=\tan ^{2} \beta\left(\left|\kappa_{\tau \mu}^{E}\right|^{2}\right) C_{\Phi} \operatorname{Br}\left(H_{k} \rightarrow \tau \tau\right),
$$




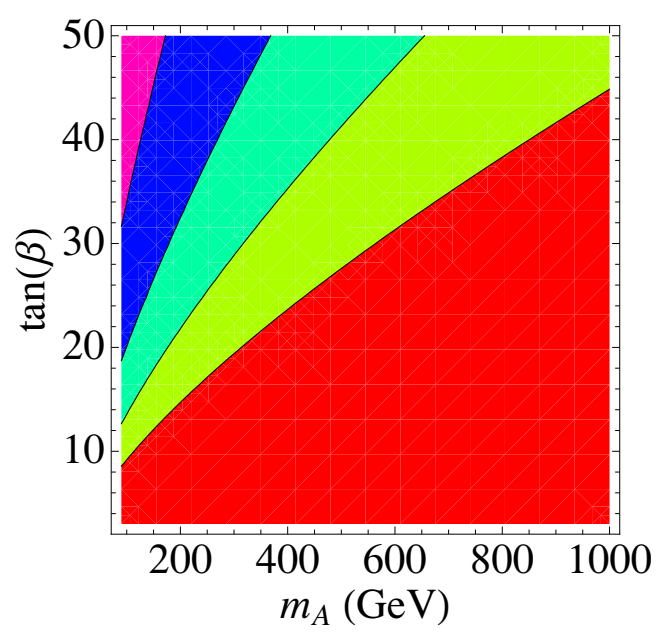

Figure 3. Branching ratio of the process $\tau \rightarrow 3 \mu$ as a function of $m_{A}(\mathrm{GeV})$ and $\tan \beta$. From left to right, the contours correspond to $\operatorname{Br}(\tau \rightarrow 3 \mu)=2.1 \times 10^{-8}, 10^{-9}, 10^{-10}, 10^{-11}$. The purple region has already been experimentally excluded [63].

where we approximated $1 / \cos ^{2} \beta \simeq \tan ^{2} \beta$. The coefficients $C_{\Phi}$ are given by:

$$
C_{h}=\left[\frac{\cos (\beta-\alpha)}{\sin \alpha}\right]^{2}, \quad C_{H}=\left[\frac{\sin (\beta-\alpha)}{\cos \alpha}\right]^{2}, \quad C_{A}=1 .
$$

\section{$5 \quad$ Results and discussion}

As discussed in section 3, in the inverse supersymmetric seesaw, Higgs-mediated contributions can lead to an enhancement of several LFV observables by as much as two orders of magnitude, compared to what is expected in the standard SUSY seesaw.

As expected from the analytical study of section $4, m_{A}$ and $\tan \beta$ are the most relevant parameters in the Higgs-mediated flavour violating processes. To better illustrate this, in figure 3 we study the dependence of $\operatorname{Br}(\tau \rightarrow 3 \mu)$ on the aforementioned parameters. We have assumed a common value for the squark masses, $m_{\widetilde{q}} \sim \mathrm{TeV}$, while for left- and righthanded sleptons we take $m_{\widetilde{\ell}} \sim 400 \mathrm{GeV}$ and $M_{\widetilde{\nu}^{c}} \sim 3 \mathrm{TeV}$ for the right handed sneutrinos. The contours correspond to different values of the branching ratios (the purple region has already been experimentally excluded). From this figure one can easily identify the regimes for $m_{A}$ and $\tan \beta$ which are associated to values of the LFV observables within reach of the present and future experiments.

In what follows, we numerically evaluate some LFV observables. Concerning the mSUGRA parameters (and instead of scanning over the parameter space), we have selected a few benchmark points [64] that already take into account the most recent LHC constraints $[65,66]$. We have also considered the case in which the GUT scale universality conditions are relaxed for the Higgs sector, i.e. scenarios of Non-Universal Higgs Masses (NUHM), as this allows to explore the impact of a light CP-odd Higgs boson. In table 1, we list the chosen points: CMSSM-A and CMSSM-B respectively correspond to the 10.2.2 and 40.1.1 benchmark points in [64], while NUHM-C is an example of a non-universal scenario. 


\begin{tabular}{|c|c|c|c|c|c|c|c|c|}
\hline Point & $\tan \beta$ & $m_{1 / 2}$ & $m_{0}$ & $m_{H_{U}}^{2}$ & $m_{H_{D}}^{2}$ & $A_{0}$ & $\mu$ & $m_{A}$ \\
\hline CMSSM-A & 10 & 550 & 225 & $(225)^{2}$ & $(225)^{2}$ & 0 & 690 & 782 \\
\hline CMSSM-B & 40 & 500 & 330 & $(330)^{2}$ & $(330)^{2}$ & -500 & 698 & 604 \\
\hline NUHM-C & 15 & 550 & 225 & $(652)^{2}$ & $-(570)^{2}$ & 0 & 478 & 150 \\
\hline
\end{tabular}

Table 1. Benchmark points used in the numerical analysis (dimensionful parameters in $\mathrm{GeV}$ ). CMSSM-A and CMSSM-B correspond to 10.2.2 and 40.1.1 benchmark points of [64].

For each point considered, the low-energy SUSY parameters were obtained using SuSpect [67]. In what concerns the evolution of the soft-breaking right-handed sneutrino masses $m_{\tilde{\nu}^{c}}^{2}$, we have assumed that the latter hardly run between the GUT scale and the low-energy one. The flavour-violating charged slepton parameters (e.g. $\left(\Delta m_{\widetilde{L}}^{2}\right)_{i j}$ or $\xi$ ), were estimated at the leading order using eq. (3.1). Concerning NUHM, we use the same value of $\xi$ as for CMSSM-A. Here, we are particularly interested to study the effect of light CP-odd Higgs boson and this naive approximation will serve our purpose. Furthermore, we use the mass insertion approximation, assuming that mixing between left and right chiral slepton states are relatively small. In computing the branching fractions and the flavour violating factor $\kappa_{i j}^{E}$ we have assumed (physical) right-handed sneutrino masses $M_{\widetilde{\nu}^{c}} \approx 3 \mathrm{TeV}$ and $\left(Y_{\nu}^{\dagger} Y_{\nu}\right)=0.7$, in agreement with low-energy neutrino data as well as other low-energy constraints, which are particularly relevant in the inverse seesaw case such as Non-Standard Neutrino Interactions bounds [68, 69]. Moreover, in our numerical analysis, we have fixed the trilinear soft breaking parameter $A_{\nu}=-500 \mathrm{GeV}$ (at the SUSY scale).

We now proceed to present our results for the flavour violating observables discussed in section 4. In table 2, we collect the values of the different branching ratios, as obtained for the considered benchmark points of table 1. We have also presented the corresponding current experimental bounds and future sensitivity.

From table 2, one can verify that from an experimental point of view, the most promising channel in the supersymmetric inverse seesaw is $\tau \rightarrow \mu \eta$ which could be tested at the next generation of $B$ factories. The $B_{d, s}^{0} \rightarrow \mu \tau$ decay might also be interesting, but little conclusions can be drawn due to lack of information concerning the future sensitivities.

It is important to stress that the numerical results summarised in table 2 correspond to considering only Higgs-mediated contributions. In the low $\tan \beta$ regime, photon- and $Z$ penguin diagrams may induce comparable or even larger contributions to the observables, and potentially enhance the branching fractions. Thus, the results for small $\tan \beta$ should be interpreted as conservative estimates, representing only partial contributions. For large $\tan \beta$ values, Higgs penguins do indeed provide the leading contributions. Comparing our results with those obtained for a type I SUSY seesaw at high scales (or even with a TeV scale SUSY seesaw), we find a large enhancement of the branching fractions in the inverse seesaw framework.

Another interesting property of the Higgs-mediated processes is that the corresponding amplitude strongly depends on the chirality of the heaviest lepton (be it the decaying lepton, or the heaviest lepton produced in $B$ decays). Considering the decays of a left- 


\begin{tabular}{|c|c|c|c|c|c|}
\hline LFV Process & Present Bound & Future Sensitivity & CMSSM-A & CMSSM-B & NUHM-C \\
\hline$\tau \rightarrow \mu \mu \mu$ & $2.1 \times 10^{-8}[63]$ & $8.2 \times 10^{-10}[73]$ & $1.4 \times 10^{-15}$ & $3.9 \times 10^{-11}$ & $8.0 \times 10^{-12}$ \\
$\tau^{-} \rightarrow e^{-} \mu^{+} \mu^{-}$ & $2.7 \times 10^{-8}[63]$ & $\sim 10^{-10}[73]$ & $1.4 \times 10^{-15}$ & $3.4 \times 10^{-11}$ & $8.0 \times 10^{-12}$ \\
$\tau \rightarrow e e e$ & $2.7 \times 10^{-8}[63]$ & $2.3 \times 10^{-10}[73]$ & $3.2 \times 10^{-20}$ & $9.2 \times 10^{-16}$ & $1.9 \times 10^{-16}$ \\
$\mu \rightarrow e e e$ & $1.0 \times 10^{-12}[1]$ & & $6.3 \times 10^{-22}$ & $1.5 \times 10^{-17}$ & $3.7 \times 10^{-18}$ \\
$\tau \rightarrow \mu \eta$ & $2.3 \times 10^{-8}[70]$ & $\sim 10^{-10}[73]$ & $8.0 \times 10^{-15}$ & $3.3 \times 10^{-10}$ & $4.6 \times 10^{-11}$ \\
$\tau \rightarrow \mu \eta^{\prime}$ & $3.8 \times 10^{-8}[70]$ & $\sim 10^{-10}[73]$ & $4.3 \times 10^{-16}$ & $1.1 \times 10^{-10}$ & $3.1 \times 10^{-12}$ \\
$\tau \rightarrow \mu \pi^{0}$ & $2.2 \times 10^{-8}[70]$ & $\sim 10^{-10}[73]$ & $1.8 \times 10^{-17}$ & $8.5 \times 10^{-13}$ & $1.0 \times 10^{-13}$ \\
$B_{d}^{0} \rightarrow \mu \tau$ & $2.2 \times 10^{-5}[71]$ & & $2.7 \times 10^{-15}$ & $8.5 \times 10^{-10}$ & $2.7 \times 10^{-11}$ \\
$B_{d}^{0} \rightarrow e \mu$ & $6.4 \times 10^{-8}[72]$ & $1.6 \times 10^{-8}[74]$ & $1.2 \times 10^{-17}$ & $3.1 \times 10^{-12}$ & $1.2 \times 10^{-13}$ \\
$B_{s}^{0} \rightarrow \mu \tau$ & & & $7.7 \times 10^{-14}$ & $2.5 \times 10^{-8}$ & $7.8 \times 10^{-10}$ \\
$B_{s}^{0} \rightarrow e \mu$ & $2.0 \times 10^{-7}[72]$ & $6.5 \times 10^{-8}[74]$ & $3.4 \times 10^{-16}$ & $8.9 \times 10^{-11}$ & $3.4 \times 10^{-12}$ \\
$h \rightarrow \mu \tau$ & & & $1.3 \times 10^{-8}$ & $2.6 \times 10^{-7}$ & $2.3 \times 10^{-6}$ \\
$A, H \rightarrow \mu \tau$ & & & $3.4 \times 10^{-6}$ & $1.3 \times 10^{-4}$ & $5.0 \times 10^{-6}$ \\
\hline
\end{tabular}

Table 2. Higgs-mediated contributions to the branching ratios of several lepton flavour violating processes, for the different benchmark points of table 1 . We also present the current experimental bounds and future sensitivities for the LFV observables.

handed lepton $\ell_{L}^{i} \rightarrow \ell_{R}^{j} X$, one finds that the corresponding branching ratios would be suppressed by a factor $\frac{m_{\ell j}^{2}}{m_{\ell^{i}}^{2}}$ compared to those of the right-handed lepton $\ell_{R}^{i} \rightarrow \ell_{L}^{j} X$. This can induce an asymmetry that potentially allows to identify if Higgs mediation is the dominant contribution to the LFV observables. Furthermore this asymmetry would be more pronounced in the inverse-seesaw framework.

Due to its strong enhancement of the Higgs-penguin contributions, if realised in Nature, the inverse seesaw offers a unique framework to test Higgs effects in LFV processes. In fact, and as discussed in [47, 48], if photon penguins provide the dominant contribution to both $\operatorname{Br}(\tau \rightarrow 3 \mu)$ and $\operatorname{Br}(\tau \rightarrow \mu \gamma)$, then the latter observables are strongly correlated, $\frac{\operatorname{Br}(\tau \rightarrow 3 \mu)}{\operatorname{Br}(\tau \rightarrow \mu \gamma)} \sim 0.003$ (see $\left.[47,48]\right)$. On the other hand, if the dominant contribution to the three-body decays arises from Higgs penguins, the correlation no longer holds, and the latter ratio can be significantly enhanced. This would be the case of the present framework.

\section{Conclusions}

If observed, charged lepton flavour violation clearly signals the presence of new physics. In this work, we have studied Higgs-mediated LFV processes in the framework of the supersymmetric inverse seesaw. TeV scale right-handed neutrinos (and hence light righthanded sneutrinos) offer the possibility to enhance the Higgs-mediated contributions to several LFV processes. As shown in this work, in the inverse SUSY seesaw, LFV branching ratios can be enhanced by as much as two orders of magnitude when compared to the standard (type I) SUSY seesaw. 


\section{Acknowledgments}

The authors are thankful to A. Vicente for many enlightening discussions. D.D. acknowledges financial support from the CNRS. This work has been partly done under the ANR project CPV-LFV-LHC NT09-508531.

Open Access. This article is distributed under the terms of the Creative Commons Attribution License which permits any use, distribution and reproduction in any medium, provided the original author(s) and source are credited.

\section{References}

[1] SINDRUM collaboration, U. Bellgardt et al., Search for the decay $\mu^{+} \rightarrow e^{+} e^{+} e^{-}$, Nucl. Phys. B 299 (1988) 1 [inSPIRE].

[2] MEGA collaboration, M.L. Brooks et al., New limit for the family number nonconserving decay $\mu^{+} \rightarrow e^{+} \gamma$, Phys. Rev. Lett. 83 (1999) 1521 [hep-ex/9905013] [INSPIRE].

[3] J. Aysto et al., Physics with low-energy muons at a neutrino factory complex, hep-ph/0109217 [INSPIRE].

[4] BABAR collaboration, J.P. Lees et al., Limits on tau lepton-flavor violating decays in three charged leptons, Phys. Rev. D 81 (2010) 111101 [arXiv:1002.4550] [inSPIRE].

[5] SuperkekB Physics Working Group collaboration, A.G. Akeroyd et al., Physics at super $B$ factory, hep-ex/0406071 [INSPIRE].

[6] BABAR collaboration, B. Aubert et al., Search for lepton flavor violation in the decay $\tau \rightarrow \mu \gamma$, Phys. Rev. Lett. 95 (2005) 041802 [hep-ex/0502032] [InSPIRE].

[7] BABAR collaboration, B. Aubert et al., Search for lepton flavor violation in the decay $\tau^{ \pm} \rightarrow e^{ \pm} \gamma$, Phys. Rev. Lett. 96 (2006) 041801 [hep-ex/0508012] [INSPIRE].

[8] Y. Kuno, PRISM/PRIME, Nucl. Phys. Proc. Suppl. 149 (2005) 376 [inSPIRE].

[9] MEG collaboration, S. Ritt, Status of the MEG expriment $\mu \rightarrow e \gamma$, Nucl. Phys. Proc. Suppl. 162 (2006) 279 [InSPIRE].

[10] SuperB collaboration, M. Bona et al., SuperB: a high-luminosity asymmetric $e^{+} e^{-}$super flavor factory. Conceptual design report, arXiv:0709.0451 [INSPIRE].

[11] Belle collaboration, K. Hayasaka et al., New search for $\tau \rightarrow \mu \gamma$ and $\tau \rightarrow$ e $\gamma$ decays at Belle, Phys. Lett. B 666 (2008) 16 [arXiv:0705. 0650] [INSPIRE].

[12] MEG collaboration, O.A. Kiselev, Positron spectrometer of MEG experiment at PSI, Nucl. Instrum. Meth. A 604 (2009) 304 [INSPIRE].

[13] PRISM/PRIME Group collaboration, Y. Kuno et al., Letter of intent. An experimental search for a $\mu-e$ conversion at sensitivity of the order of $10^{-18}$ with a highly intense muon source: PRISM, unpublished.

[14] P. Minkowski, $\mu \rightarrow e \gamma$ at a rate of one out of 1-billion muon decays?, Phys. Lett. B 67 (1977) 421 [INSPIRE].

[15] M. Gell-Mann, P. Ramond and R. Slansky, Complex spinors and unified theories, in Supergravity, P. Van Nieuwenhuizen and D.Z. Freedman eds., North-Holland, Amsterdam Netherlands (1979), pg. 315 [INSPIRE]. 
[16] T. Yanagida, Horizontal symmetry and masses of neutrinos, in Proceedings of the Workshop on the Baryon Number of the Universe and Unified Theories, Tsukuba Japan, 13-14 Feb 1979, O. Sawada and A. Sugamoto eds., pg. 95 [INSPIRE].

[17] S.L. Glashow, The future of elementary particle physics, in Proceedings of the 1979 Cargèse Summer Institute on Quarks and Leptons, M. Lévy et al. eds., Plenum Press, New York U.S.A. (1980), pg. 687 [INSPIRE].

[18] R.N. Mohapatra and G. Senjanović, Neutrino mass and spontaneous parity violation, Phys. Rev. Lett. 44 (1980) 912 [INSPIRE].

[19] R. Barbieri, D.V. Nanopoulos, G. Morchio and F. Strocchi, Neutrino masses in grand unified theories, Phys. Lett. B 90 (1980) 91 [inSPIRE].

[20] R.E. Marshak and R.N. Mohapatra, Selection rules for baryon number nonconservation in gauge models, talk given at Orbis Scientiae, Coral Gables U.S.A., 14-17 Jan 1980, VPI-HEP-80-02 [INSPIRE].

[21] T.P. Cheng and L.-F. Li, Neutrino masses, mixings and oscillations in $\mathrm{SU}(2) \times \mathrm{U}(1)$ models of electroweak interactions, Phys. Rev. D 22 (1980) 2860 [InSPIRE].

[22] M. Magg and C. Wetterich, Neutrino mass problem and gauge hierarchy, Phys. Lett. B 94 (1980) 61 [INSPIRE].

[23] G. Lazarides, Q. Shafi and C. Wetterich, Proton lifetime and fermion masses in an $\mathrm{SO}(10)$ model, Nucl. Phys. B 181 (1981) 287 [INSPIRE].

[24] J. Schechter and J.W.F. Valle, Neutrino masses in $\mathrm{SU}(2) \times \mathrm{U}(1)$ theories, Phys. Rev. D 22 (1980) 2227 [INSPIRE].

[25] R.N. Mohapatra and G. Senjanović, Neutrino masses and mixings in gauge models with spontaneous parity violation, Phys. Rev. D 23 (1981) 165 [INSPIRE].

[26] E. Ma, Pathways to naturally small neutrino masses, Phys. Rev. Lett. 81 (1998) 1171 [hep-ph/9805219] [INSPIRE].

[27] R. Foot, H. Lew, X.G. He and G.C. Joshi, Seesaw neutrino masses induced by a triplet of leptons, Z. Phys. C 44 (1989) 441 [INSPIRE].

[28] S.F. King, Atmospheric and solar neutrinos with a heavy singlet, Phys. Lett. B 439 (1998) 350 [hep-ph/9806440] [INSPIRE].

[29] S. Davidson and S.F. King, Bimaximal neutrino mixing in the MSSM with a single right-handed neutrino, Phys. Lett. B 445 (1998) 191 [hep-ph/9808296] [INSPIRE].

[30] R.N. Mohapatra and J.W.F. Valle, Neutrino mass and baryon number nonconservation in superstring models, Phys. Rev. D 34 (1986) 1642 [InSPIRE].

[31] G. 't Hooft, Naturalness, chiral symmetry, and spontaneous chiral symmetry breaking, lecture given at Cargèse Summer Institute: Recent Developments in Gauge Theories, Cargèse France, 26 Aug-8 Sep 1979, NATO Adv. Study Inst. Ser. B Phys. 59 (1980) 135, Plenum Press, New York U.S.A. [INSPIRE].

[32] F. Deppisch and J.W.F. Valle, Enhanced lepton flavor violation in the supersymmetric inverse seesaw model, Phys. Rev. D 72 (2005) 036001 [hep-ph/0406040] [INSPIRE].

[33] F. Deppisch, T.S. Kosmas and J.W.F. Valle, Enhanced $\mu^{-}-e^{-}$conversion in nuclei in the inverse seesaw model, Nucl. Phys. B 752 (2006) 80 [hep-ph/0512360] [INSPIRE]. 
[34] J. Garayoa, M.C. Gonzalez-Garcia and N. Rius, Soft leptogenesis in the inverse seesaw model, JHEP 02 (2007) 021 [hep-ph/0611311] [INSPIRE].

[35] C. Arina, F. Bazzocchi, N. Fornengo, J.C. Romao and J.W.F. Valle, Minimal supergravity sneutrino dark matter and inverse seesaw neutrino masses, Phys. Rev. Lett. 101 (2008) 161802 [arXiv:0806.3225] [INSPIRE].

[36] E. Ma, Radiative inverse seesaw mechanism for nonzero neutrino mass, Phys. Rev. D 80 (2009) 013013 [arXiv: 0904.4450] [InSPIRE].

[37] P.S.B. Dev and R.N. Mohapatra, TeV scale inverse seesaw in $\mathrm{SO}(10)$ and leptonic non-unitarity effects, Phys. Rev. D 81 (2010) 013001 [arXiv:0910.3924] [inSPIRE].

[38] M. Malinsky, T. Ohlsson, Z.-z. Xing and H. Zhang, Non-unitary neutrino mixing and CP-violation in the minimal inverse seesaw model, Phys. Lett. B 679 (2009) 242 [arXiv: 0905.2889] [INSPIRE].

[39] F. Bazzocchi, D.G. Cerdeno, C. Muñoz and J.W.F. Valle, Calculable inverse-seesaw neutrino masses in supersymmetry, Phys. Rev. D 81 (2010) 051701 [arXiv:0907.1262] [INSPIRE].

[40] M. Hirsch, T. Kernreiter, J.C. Romao and A. Villanova del Moral, Minimal supersymmetric inverse seesaw: neutrino masses, lepton flavour violation and LHC phenomenology, JHEP 01 (2010) 103 [arXiv:0910.2435] [INSPIRE].

[41] F. Bazzocchi, Minimal dynamical inverse see saw, Phys. Rev. D 83 (2011) 093009 [arXiv: 1011.6299] [INSPIRE].

[42] J. Bernabeu, A. Santamaria, J. Vidal, A. Mendez and J.W.F. Valle, Lepton flavor nonconservation at high-energies in a superstring inspired standard model, Phys. Lett. B 187 (1987) 303 [INSPIRE].

[43] G.C. Branco, M.N. Rebelo and J.W.F. Valle, Leptonic CP-violation with massless neutrinos, Phys. Lett. B 225 (1989) 385 [inSPIRE].

[44] N. Rius and J.W.F. Valle, Leptonic CP-violating asymmetries in Z0 decays, Phys. Lett. B 246 (1990) 249 [INSPIRE].

[45] M.C. Gonzalez-Garcia and J.W.F. Valle, Enhanced lepton flavor violation with massless neutrinos: a study of muon and tau decays, Mod. Phys. Lett. A 7 (1992) 477 [INSPIRE].

[46] F. Borzumati and A. Masiero, Large muon and electron number violations in supergravity theories, Phys. Rev. Lett. 57 (1986) 961 [INSPIRE].

[47] J. Hisano, T. Moroi, K. Tobe and M. Yamaguchi, Lepton flavor violation via right-handed neutrino Yukawa couplings in supersymmetric standard model, Phys. Rev. D 53 (1996) 2442 [hep-ph/9510309] [INSPIRE].

[48] J. Hisano and D. Nomura, Solar and atmospheric neutrino oscillations and lepton flavor violation in supersymmetric models with the right-handed neutrinos, Phys. Rev. D 59 (1999) 116005 [hep-ph/9810479] [INSPIRE].

[49] L.J. Hall, R. Rattazzi and U. Sarid, The top quark mass in supersymmetric $\mathrm{SO}(10)$ unification, Phys. Rev. D 50 (1994) 7048 [hep-ph/9306309] [INSPIRE].

[50] S.R. Choudhury and N. Gaur, Dileptonic decay of $B_{s}$ meson in SUSY models with large $\tan \beta$, Phys. Lett. B 451 (1999) 86 [hep-ph/9810307] [INSPIRE].

[51] K.S. Babu and C.F. Kolda, Higgs mediated $B^{0} \rightarrow \mu^{+} \mu^{-}$in minimal supersymmetry, Phys. Rev. Lett. 84 (2000) 228 [hep-ph/9909476] [INSPIRE]. 
[52] G. Isidori and A. Retico, Scalar flavor changing neutral currents in the large $\tan \beta$ limit, JHEP 11 (2001) 001 [hep-ph/0110121] [INSPIRE].

[53] G. Isidori and A. Retico, $B_{s, d} \rightarrow \ell^{+} \ell^{-}$and $K_{L} \rightarrow \ell^{+} \ell^{-}$in SUSY models with nonminimal sources of flavor mixing, JHEP 09 (2002) 063 [hep-ph/0208159] [INSPIRE].

[54] A.J. Buras, P.H. Chankowski, J. Rosiek and L. Slawianowska, $\Delta M_{s} / \Delta M_{d}, \sin 2 \beta$ and the angle $\gamma$ in the presence of new $\Delta F=2$ operators, Nucl. Phys. B 619 (2001) 434 [hep-ph/0107048] [INSPIRE].

[55] A.J. Buras, P.H. Chankowski, J. Rosiek and L. Slawianowska, Correlation between $\Delta M_{s}$ and $B_{s, d}^{0} \rightarrow \mu^{+} \mu^{-}$in supersymmetry at large $\tan \beta$, Phys. Lett. B 546 (2002) 96 [hep-ph/0207241] [INSPIRE].

[56] G. D'Ambrosio, G.F. Giudice, G. Isidori and A. Strumia, Minimal flavor violation: an effective field theory approach, Nucl. Phys. B 645 (2002) 155 [hep-ph/0207036] [INSPIRE].

[57] A. Dedes, J.R. Ellis and M. Raidal, Higgs mediated $B_{s, d}^{0} \rightarrow \mu \tau$, e $\tau$ and $\tau \rightarrow 3 \mu$, e $\mu \mu$ decays in supersymmetric seesaw models, Phys. Lett. B 549 (2002) 159 [hep-ph/0209207] [INSPIRE].

[58] K.S. Babu and C. Kolda, Higgs mediated $\tau \rightarrow 3 \mu$ in the supersymmetric seesaw model, Phys. Rev. Lett. 89 (2002) 241802 [hep-ph/0206310] [InSPIRE].

[59] M. Sher, $\tau \rightarrow \mu \eta$ in supersymmetric models, Phys. Rev. D 66 (2002) 057301 [hep-ph/0207136] [INSPIRE].

[60] A. Brignole and A. Rossi, Anatomy and phenomenology of $\mu-\tau$ lepton flavor violation in the MSSM, Nucl. Phys. B 701 (2004) 3 [hep-ph/0404211] [INSPIRE].

[61] C.W. Bernard, Heavy quark physics on the lattice, Nucl. Phys. Proc. Suppl. 94 (2001) 159 [hep-lat/0011064] [INSPIRE].

[62] A. Brignole and A. Rossi, Lepton flavor violating decays of supersymmetric Higgs bosons, Phys. Lett. B 566 (2003) 217 [hep-ph/0304081] [INSPIRE].

[63] K. Hayasaka et al., Search for lepton flavor violating $\tau$ decays into three leptons with 719 million produced $\tau^{+} \tau^{-}$pairs, Phys. Lett. B 687 (2010) 139 [arXiv:1001.3221] [INSPIRE].

[64] S.S. AbdusSalam et al., Benchmark models, planes, lines and points for future SUSY searches at the LHC, Eur. Phys. J. C 71 (2011) 1835 [arXiv:1109. 3859] [InSPIRE].

[65] CMS collaboration, S. Chatrchyan et al., Search for supersymmetry in pp collisions at $\sqrt{s}=7 \mathrm{TeV}$ in events with a single lepton, jets and missing transverse momentum, JHEP 08 (2011) 156 [arXiv:1107.1870] [INSPIRE].

[66] ATLAS collaboration, G. Aad et al., Search for supersymmetry in final states with jets, missing transverse momentum and one isolated lepton in $\sqrt{s}=7 \mathrm{TeV}$ pp collisions using $1 \mathrm{fb}^{-1}$ of ATLAS data, Phys. Rev. D 85 (2012) 012006 [arXiv:1109.6606] [INSPIRE].

[67] A. Djouadi, J.-L. Kneur and G. Moultaka, SuSpect: a Fortran code for the supersymmetric and Higgs particle spectrum in the MSSM, Comput. Phys. Commun. 176 (2007) 426 [hep-ph/0211331] [INSPIRE].

[68] S. Antusch, C. Biggio, E. Fernandez-Martinez, M.B. Gavela and J. Lopez-Pavon, Unitarity of the leptonic mixing matrix, JHEP 10 (2006) 084 [hep-ph/0607020] [INSPIRE].

[69] S. Antusch, J.P. Baumann and E. Fernandez-Martinez, Non-standard neutrino interactions with matter from physics beyond the standard model, Nucl. Phys. B $\mathbf{8 1 0}$ (2009) 369 [arXiv: 0807.1003] [INSPIRE]. 
[70] Belle collaboration, K. Hayasaka, Search for lepton-flavor-violating $\tau$ decay and lepton-number-violation B decay at Belle, PoS(ICHEP 2010) 241 [arXiv:1011.6474] [INSPIRE].

[71] BABAR collaboration, B. Aubert et al., Searches for the decays $B^{0} \rightarrow \ell^{ \pm} \tau^{\mp}$ and $B^{+} \rightarrow \ell^{+} \nu$ $(l=e, \mu)$ using hadronic tag reconstruction, Phys. Rev. D 77 (2008) 091104 [arXiv:0801.0697] [INSPIRE].

[72] CDF collaboration, T. Aaltonen et al., Search for the decays $B_{(s)}^{0} \rightarrow e^{+} \mu^{-}$and $B_{(s)}^{0} \rightarrow e^{+} e^{-}$ in CDF Run II, Phys. Rev. Lett. 102 (2009) 201801 [arXiv:0901.3803] [INSPIRE].

[73] SuperB collaboration, B. O'Leary et al., SuperB progress reports - physics, arXiv: 1008.1541 [INSPIRE].

[74] W. Bonivento and N. Serra, LHCb sensitivity to the $L F V B B_{d, s}^{0} \rightarrow e^{ \pm} \mu^{\mp}$ decays, CERN-LHCB-2007-028 [inSPIRE]. 